\title{
Neuere Entwicklungen der landwirtschaftlichen Nutzung im Walliser Berggebiet
}

\section{Résumé}

Les villages de montagne subirent un exode permanent lors de l'industrialisation et de l'urbanisation des centres de la vallée principale du Valais. La diminution de l'élevage du bétail et la mise en friche de vastes terrains agricoles ont provoqué des problèmes d'esthétique et de sauvegarde du paysage. L'Office d'économie montagnarde du Département de l'intérieur du Canton du Valais developpa un programme en vue d'améliorer l'économie agricole de la montagne; certaines mesures préconisées par ce dernier ont été réalisées à ce jour.

En 1974, les Chambres de l'Agriculture des Cantons de Vaud et de Fribourg lancèrent une action à double but: pacage de jeune bétail provenant de la plaine, ce qui permet d'une part d'éviter une perte de fourrage et d'autre part de lutter contre l'abandon des terrains montagnards. Ce système d'exploitation serait en mesure d'assurer, adjoint aux formes traditionnelles de l'agriculture, une lutte victorieuse contre une continuation de la mise en friche du paysage alpin.

Im Laufe der letzten zwanzig Jahre zeitigte die Wirtschaftspolitik der Walliser Regierung Erfolge in der Steigerung der wirtschaftlichen Aktivität im Haupttal. Die sich entwickelnden Zentren von Brig bis Martigny vermochten laufend neue Arbeitsplätze im sekundären und tertiären Sektor anzubieten. Eine stark intensivierte Landwirtschaft mit Früchte- und Gemüsekulturen im Talboden, mit Rebbau an den Sonnenhängen und bis in die Sohle ermöglichte einerseits rein landwirtschaftliche, andererseits doppelberufliche Erwerbstätigkeit. Während sich die Pendlereinzugsgebiete früher auf talnahe Hanglagen konzentrierten, reichen sie heute dank der verbesserten privaten Mobilität bis weit in die Seitentäler hinein. Der in den fünfziger Jahren einsetzende Exodus aus den Bergdörfern und der Zerfall der traditionellen Berglandwirtschaft haben sich bis in die jüngste Zeit noch verstärkt.

DUMONT hatte 1954 die im Val d'Anniviers praktizierte Landwirtschaft als hochgradig unökonomisch qualifiziert bezüglich Arbeitswege, Gebäudekosten, Parzellierung und Größe der Betriebe sowie der geländebedingt geringen Mechanisierungsmöglichkeiten. $\mathrm{Er}$ schlug eine großräumige Transhumanz mit Sömmerung von Unterlandvieh im Berggebiet und Winter- fütterung bei Stallhaltung im Schweizerischen Mittelland vor. Das wäre gleichbedeutend gewesen mit einer Degradierung der Bergdörfer zu reinen Sommerresidenzen für Viehhüter aus dem Unterland.

Die Implantation von Kleinindustrien in den Seitentälern (vgl. BUGMANN 1974) vermochte den Zerfall der Berglandwirtschaft nur unwesentlich aufzuhalten. Auch die Entwicklung des Tourismus in den Bergdörfern bewirkte vorerst nicht die erwünschte Umkehr des Entleerungstrendes (Beispiele bei BÄR 1969 , GARIN 1971, BRIDEL 1971, BUGMANN 1972).

Die Strukturkrise in den Bergdörfern bezog sich im wesentlichen auf folgende Bereiche (SURBER e.a. 1973, BUGMANN 1974):

a) Rückgang der Viehhaltung

b) Aufgabe der Bewässerungswirtschaft, Brachfallen von Mähwiesen und Weideland

c) Fast vollständiges Verschwinden des Ackerbaus auf den traditionellen Roggenzelgen

d) Probleme der Landschaftsästhetik, des Landschaftsund Siedlungsschutzes

e) Mangel an erwerbsfähigen Einheimischen beim Umstellen der Bergdörfer auf den Tourismus.

Das Walliser Landwirtschaftsdepartement hat zur Verbesserung der Berglandwirtschaft folgenden Maßnahmenkatalog entwickelt (PICCOT 1975):

1. Restrukturierung der über eine ausreichende Produktionsbasis verfügenden Betriebe durch:

a) Vergrößerung der Betriebsfläche mittels Kauf oder Pacht von Nutzland,

b) Modernisierung landwirtschaftlicher Gebäude und maschineller Ausrüstung

2. Bau von kooperativ bewirtschafteten Gemeinschaftsställen im Hinblick auf rationellere winterliche Stallfütterung

3. Güterzusammenlegungen und Betriebssanierungen

4. Intensivierte landwirtschaftliche Berufsbildung

5. Einführung von Formen intensivierter Tierzucht wie Geflügelhaltung und Schweinezucht

6. Förderung von Spezialkulturen in klimatisch geeigneten Regionen (Erdbeeren, Himbeeren, Kartoffelsaatgut, Gemüse, Weinbau und Obstkulturen)

PD Dr. Erich Bugmann, Geographisches Institut,

ETH Zentrum, 8092 Zürich. 
7. Verbesserung der Gras- und Heugewinnung und der Alpweiden

8. Verbesserung des Rindviehbestandes durch Zuchtselektion

9. Rationelle Vermarktung von Milchprodukten (z. B. für Raclette-Käse), Zucht- und Schlachtvieh

Zusätzlich wird mit Bundessubventionen an Viehhalter im Berggebiet und sozialen Ausgleichszahlungen gerechnet.

Die langfristigen Aussichten für die Wahrung eines Minimalbestandes von landwirtschaftlichen Individual- und Kollektivbetrieben sind auf der Basis dieses Programmes durchaus gut. Es hat sich aber während der laufenden Umstrukturierungsphase gezeigt, daß Nutzflächen in Steillagen, welche früher von Hand gemäht oder mit der Hacke bearbeitet wurden, maschinell nicht genutzt werden können und deshalb brachfallen müssen. Eine Beweidung solcher Flächen ist erst interessant, wenn die Kapazität der Alpweiden ausgelastet ist (in St. Luc im Val d'Anniviers liegt sie zur Zeit bei $50 \%$ ). Diese Entwicklung ist in Bergdörfern mit bedeutenden touristischen Funktionen aus Gründen der Landschaftsästhetik sehr unerwünscht. Die Gemeinde St. Luc erzwang im Jahre 1972 die Pflege von Sozial- und Grenzertragsbrache im Dorfgebiet mit feuerpolizeilichen Argumenten (BUGMANN 1974); das auf Kosten der Grundeigentümer geschnittene verdorrte Gras wanderte auf die kommunale Kehrichtdeponie. Diese Zwangsmaßnahme hat indessen nicht weiter Schule gemacht, ist sie doch durch ein neues Konzept abgelöst worden.

Ein Landwirt aus Chalet à Gobet ob Lausanne hatte im Jahre 1973 erfolgreich Jungvieh im Unterwallis gesömmert. Nach weiteren Versuchen im Jahre 1974 wurde die Aktion "pacage de jeune bétail de la pleine» durch Zusammenarbeit zwischen den Chambres d'agricultures der Kantone Waadt und Freiburg und dem Office d'économie montagnarde des Kantons Wallis auf 800 Haupt Jungvieh ausgedehnt, die im Jahre 1975 in den Gemeinden Valsorey, Hérémence, St. Martin, Ayent, Ayer und St. Luc gesömmert werden konnten. Der Kanton Wallis subventionierte die Umzäunung fester Weidegebiete in ungenutztem offenem Land; die Berggemeinden übernahmen die Betreuung des zu sömmernden Viehs gegen eine Entschädigung von einem Franken pro Haupt und Sömmerungstag.

\section{Perspektiven einer zukünftigen Flächennutzung am Beispiel von St. Luc}

(Nutzungskartierung, Abb. 1)

Außer den im Dorfgebiet umzäunten Weideflächen wurden die früheren Mayens La Barme (westlich an das skizzierte Gebiet anschließend) und Le Gilou (zwei Kilometer südwestlich des Dorfes gelegen) für die Sömmerung des Unterlandviehs vorbereitet. Auf Ausschreibung der Gemeinde meldeten sich aus dem Dorf 22 Helfer für die Betreuung des Pensionsviehs; das Land wurde ohne Entschädigungsansprüche zur Verfügung gestellt. Der Hauptteil der Betreuungsarbeiten konnte über das Wochenende oder nach Feierabend erledigt werden: Eintreiben der Jungrinder in neue Weiden, Umstellen der elektrischen Viehhüter, Einleiten von Trinkwasser aus den Hauptwasserleiten (Bisses) des Dorfes. Das Unterlandvieh wurde auf den 1. Juli angeliefert und am 15. Oktober mit Viehtransportwagen wieder abgeholt (vgl. Titelbild). Die bereitgestellte Weidekapazität wurde von den 123 Stück Pensionsvieh nicht voll beansprucht. Die Beweidung selbst recht steiler Partien war vorzüglich, die Herde blieb von ins Gewicht fallenden Unfällen verschont. Schwierigkeiten in der Trinkwasserbeschaffung stellten sich lediglich im Mayen Gilou ein. Die Landschaftspflege aus der Sicht der touristischen Wünschbarkeit wurde ideal erreicht. Das im engeren Dorfgebiet gehaltene Vieh bildete - in Ergänzung zu der gealpten 120köpfigen Ehringerherde - während der ganzen Sommersaison eine Attraktion ersten Ranges für die Feriengäste. Sollte diese Form der Jungviehsömmerung sich als feste Nutzungsart etablieren, zeichneten sich folgende Tendenzen in der Flächennutzung ab (Abb. 1):

\section{A. Private oder korporative Haltung des traditionellen Ehringerviehs:}

Nutzung von Heuwiesen (z. B. bewässert) und Weiden im Dorfgebiet, korporative Alpung im Sommer, Winterfütterung in Gemeinschaftsställen. Der Viehbestand wird limitiert durch die Produktionskapazität der Heuwiesen. Deren Fläche mißt rund 45 Hektaren. Bei einer mit Einschluß der Bewässerungsmöglichkeit grob veranschlagten Tragfähigkeit des Heuwieslandes von 1,2 Großvieheinheiten pro Hektare könnten rund 60 Stück Großvieh gehalten werden, das heißt, daß der 1975 von den drei Bauern in St. Luc gehaltene Groß- 
Abb. 1: Flächennutzung in St. Luc im Sommer 1975. 1 = Dorfzentrum, Gärten, 2 = Steile und felsige Partien, nicht genutzt, $3=$ Kartoffelfelder, teilweise genutzt, $4=$ Gebiete der Wohnzone, Wiesen teilweise gemäht, $5=$ Mähwiesen, $6=$ Private Weiden, $7=$ Umzäunte Weiden für das Unterlandjungvieh, $8=$ «Ferme Salamin», $9=$ Kartoffel- und Roggenfelder, $10=$ Gebiete früherer Roggenzelgen, $11=$ früheres Wiesland, $12=$ Waldränder, $13=$ Ortsverbindungsstraßen

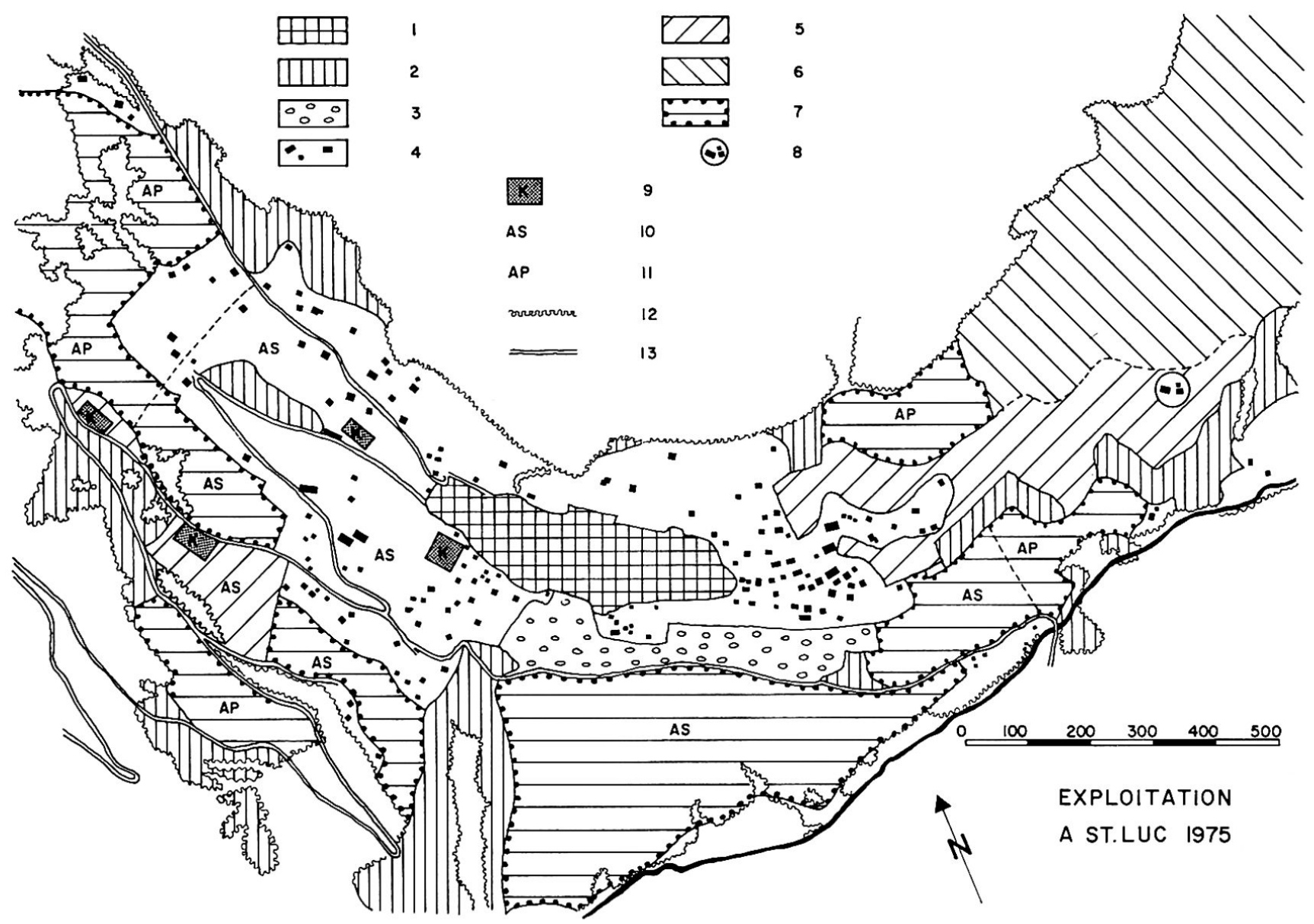

viehbestand von 20 Haupt sich wahrscheinlich verdreifachen ließe. Die aus dem Dorfgebiet bereits ausgesiedelten Ökonomiegebäude der Gebrüder Salamin («Ferme Salamin») wären als Entwicklungskern zukünftiger korporativer Nutzung denkbar.

\section{B. Kartoffel-, Gemüse- und Roggenanbau für die Selbstversorgung:}

Wegen starken Vogelfraß-Schäden sind bis 1975 die Roggenzelgen mit einer einzigen Ausnahme verschwunden. Der Anbau von Kartoffeln für den Eigenbedarf hat nach einem starken Rückgang um 1970 wieder etwas angezogen. Der Gemüseanbau hat sich in einem guten Dutzend Hausgärtchen durchgesetzt. Parallel zu einer Ernüchterung nach dem verwehten Wohlstandstaumel der Hochkonjunktur dürfte die
Besinnung auf den Wert manueller Freizeitarbeit und auf dank pedologischen und klimatischen Verhältnissen erzielbaren qualitativ hervorragenden Ernten von Kartoffeln und ausgewählten Gemüsearten zu vermehrten Umbrüchen von kleinen Ackerflächen südöstlich des Dorfes führen. Da dieses Areal für die Selbstversorgung der dörflichen Haushalte langfristig mehr als ausreichend ist, wäre es in Teilen auch als Heuwiesland nutzbar.

\section{Sömmerungsweiden für Jungvieh aus dem Unterland}

Bei voller Beweidung dürften diese Flächen für 150 bis 200 Stück Jungvieh ausreichen. Es wäre denkbar, $\mathrm{da} ß$ bei einer wieder stärkeren Ausdehnung des einheimischen Viehbestandes einige Hektaren dieser 
Areale, die wegen geringerer Geländeneigung maschinell bearbeitbar sind, zu den für den einheimischen Viehbestand nutzbaren Heuwiesen umgezont werden könnten. Im Zuge dauernder Beweidung ehemaliger Kartoffelzelgen muß weitgehende Zerstörung terrassierter Felder durch Viehtritt in Kauf genommen werden.

\section{Brachfallendes Land:}

Alle zu steilen und teilweise felsdurchsetzten Areale werden einer weiteren Nutzung entzogen bleiben. Diese Flächen sind wohl zum kleinsten Teil in die Bestände forstwirtschaftlich nutzbaren Waldes umfunktionierbar (vgl. SURBER 1973). Vielmehr ist hier mit der Entwicklung interessanter Biotop-Sukzessionen zu rechnen. Sie werden die gepflegte Erholungslandschaft bereichern und wären als Landschaftsschutzgebiete planerisch zu sichern.

Das im Wallis eingeleitete Nutzungskonzept scheint sich als ein praktikables Instrument zur Lösung des Brachlandproblems abzuzeichnen, das insbesondere für Erholungsgebiete interessante Aspekte aufweist. In ihm werden die von DUMONT (1954) suggerierten Vorschläge teilweise realisiert. Abzuklären wird in Zukunft noch sein, ob Sommerpension, zweimaligem Viehtransport und Winterfütterung im Unterland eine größere Rentabilität eigen ist als einer denkbaren Alternative mit Dauerhaltung im Berggebiet bei Winterfütterung mit Heuzufuhren aus dem Unterland. Jedenfalls ist von größter Bedeutung, daß dank eigenständiger Landwirtschaft auch in touristisch teilweise umstrukturierten Bergdörfern ein Stück überlieferter Nutzungsform durch die eingesessene Bevölkerung weitergeführt werden kann. Das bedeutet nicht nur aktive Pflege der Natur- und Kulturlandschaft; damit wird anstelle rein musealen Bewahrens von Bauformen, Gebäudetypen und dörflichen Traditionen weiterhin eine lebendige Realisierung des Gemeinschaftslebens im Rahmen der dörflichen Eigenart möglich.

\section{Zitierte Literatur}

BÄR, O. (1969): Chandolin 1968. Geographica Helvetica 24, p. 1-7.

BRIDEL, L. (1971): Spéculation ou renouveau dans les Alpes? Etudes de lettres, série III, tome 4, No 2, Université de Lausanne, p. 46-76.

BUGMANN, E. (1972): La transformation des paysages ruraux en paysages touristiques au Canton de Valais. International Geography, University of Toronto Press, p. 706-707.

BUGManN, E. (1974): St. Luc: Ein Walliser Bergdorf wird Touristenstation. Geographica Helvetica 29, p. 83-95.

DUMONT, R. (1954): Economie agricole dans le monde. $2^{\text {me }}$ édition anglaise: "Types of rural Economy», Methuen London.

GARIN, M. (1971): Un village valaisan: Pinsec. Bulletin de la Société Neuchâteloise de Géographie 54-4, No 16, p. 33-73.

PICCOT, M. (1975): L'agriculture valaisanne. Revue suisse d'agriculture, No 1, p. 1-8.

SURBER, E. e.a. (1973): Das Brachlandproblem in der Schweiz. Eidg. Versuchsanstalt für das forstliche Versuchswesen, Birmensdorf. 